\title{
Application of large-area avalanche photodiodes to energy-dispersive $x$-ray fluorescence analysis ${ }^{\dagger}$
}

\author{
L. M. P. Fernandes, J. A. M. Lopes, J. M. F. dos Santos* and C. A. N. Conde \\ Physics Department, University of Coimbra, P-3004-516 Coimbra, Portugal
}

Received 27 October 2000; Accepted 15 January 2001

\begin{abstract}
The performance of several large-area avalanche photodiodes (LAAPDs) for application to x-ray spectrometry at room temperature was investigated. Optimum detector performance was obtained for gains of about 50-70. Good energy linearity of the LAAPDs' response to x-rays was observed up to energies of about $25 \mathrm{keV}$. For this energy range the space charge effects are negligible for gains below 100-150. The energy resolutions obtained with LAAPDs can be better than those obtained with proportional counters. Our results indicate that the energy resolution of an LAAPD is better than that of a proportional counter only when the LAAPD maximum dark current is $<300 \mathrm{nA}$. For this case, minimum detectable x-ray energies of about $1 \mathrm{keV}$ are possible. Spatial non-uniformity is responsible for an absolute increase of $1-2 \%$ in energy resolution at $6 \mathrm{keV}$ when illumination of the LAAPD is broadened from $1 \mathrm{~mm}$ diameter to full area. Counting rates up to $5 \times 10^{4}$ counts $\mathrm{s}^{-1}$ are possible without significant degradation of detector performance. Copyright $\subset 2001$ John Wiley \& Sons, Ltd.
\end{abstract}

\section{INTRODUCTION}

Avalanche photodiodes (APDs) are compact, monolithic devices made of a silicon $\mathrm{p}-\mathrm{n}$ junction where the internal electric field can reach values high enough to allow electron multiplication by impact ionization. ${ }^{1-3}$ When a high voltage is applied to the APD, only a small region of the p layer, at the front of the diode, remains undepleted - the drift region (Fig. 1). The electric field in this region is low but, in the depleted region, increases with depth, reaching a maximum around the $\mathrm{p}-\mathrm{n}$ junction. An incident photon absorbed in the drift region or in the $\mathrm{p}$-depleted layer produces electron-hole pairs. The resulting electrons are accelerated towards the $\mathrm{n}^{+}$ contact and undergo avalanche multiplication owing to the high electric field around the junction. Charge gains of a few hundred are typical and increase exponentially with applied voltage, resulting in a significant improvement of the signalto-noise ratio. Detailed operation principles of this device have been discussed in the literature. ${ }^{1-3}$

Non-uniformity has been a major drawback in the manufacture of large-area APDs and has limited their applications. Recently, large-area avalanche photodiodes (LAAPDs) have been developed with improved spatial uniformity, delivering higher gains at lower bias voltages ${ }^{3}$ (Advanced Photonix, Camarillo, CA, USA; Radiation Monitoring Devices, Watertown, MA, USA). Windowless LAAPDs with extended sensitivity to UV and soft $x$-ray regions are also commercially available.

Interest in applications of LAAPDs has been increasing over the last several years. They are used mostly as optical

*Correspondence to: J. M. F. dos Santos, Physics Department, University of Coimbra, P-3004-516 Coimbra, Portugal.

${ }^{\dagger}$ Presented at the European Conference on EDXRS, Krakow, Poland, 18-23 June 2000.

Contract/grant sponsor: CERN; Contract/grant number:

CERN/P/FIS/15200/99. photodetectors coupled to scintillators for $\mathrm{x}$ - and $\gamma$-ray detection in substitution of photomultipliers ${ }^{3-5}$ (M. Moszynski, M. Kapusta, J. Zalipska, M. Balcerzyk, D. Wolski, M. Szawlowski and W. Klamra, presented at the 1999 IEEE Nuclear Science Symposium, 24-30 October, Seattle, WA, USA; M. Kapusta, M. Moszynski, M. Balcerzyk, K. Lesniewski, and M.Szawlowski, presented at the 1999 IEEE Nuclear Science Symposium, 24-30 October, Seattle, WA, USA). The feasibility of using LAAPDs as x-ray detectors has been demonstrated ${ }^{2}$ (M. Moszynski, M. Szawlowski, M. Kapusta, M. Balcerzyk and D. Wolski, presented at the 1999 IEEE Nuclear Science Symposium, 24-30 October, Seattle, WA, USA). Since the useful thickness for $\mathrm{x}$ ray detection is only a few tens of micrometers, the LAAPD detection efficiency decreases rapidly for energies above $5 \mathrm{keV}$, being about $45,25,8$ and $4 \%$ for $8,10,15$ and $20 \mathrm{keV}$, respectively. ${ }^{1,6}$ Although their use as x-ray detectors in the $0.5-20 \mathrm{keV}$ range has been suggested $^{6-8}$ (M. Moszynski, M. Szawlowski, M. Kapusta, M. Balcerzyk and D. Wolski, presented at the 1999 IEEE Nuclear Science Symposium, 24-30 October, Seattle, WA, USA), low-energy $x$-ray detection techniques with APDs were mainly developed to measure charge-carrier properties of the device, using x-rays as a reference for light measurements ${ }^{3,6,8,9}$ (M. Moszynski, M. Szawlowski, M. Kapusta, M. Balcerzyk and D. Wolski, presented at the 1999 IEEE Nuclear Science Symposium, 24-30 October, Seattle, WA, USA).

In this work, the performance of LAAPDs, working at room temperature, for energy-dispersive $x$-ray fluorescence analysis is presented. Energy linearity, energy resolution and count-rate capability were studied as a function of x-ray energy. The effects of detector dark current and gain on the minimum detectable $x$-ray energy and on the detector energy 


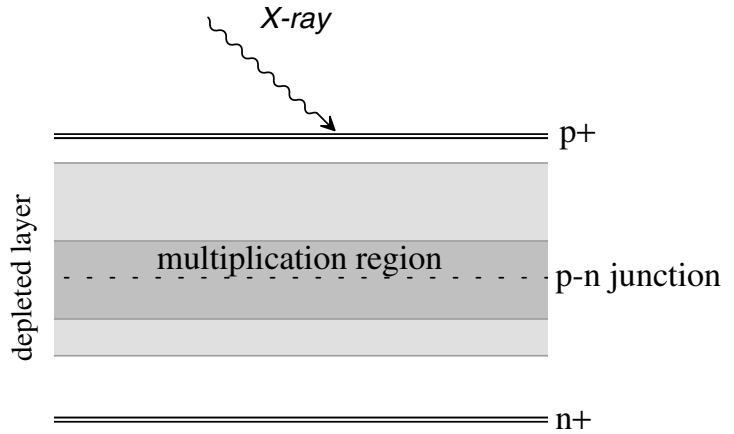

Figure 1. Schematic diagram of typical avalanche photodiode section.

resolution are discussed. Space charge effects for high gains and high-energy $x$-rays were investigated.

\section{EXPERIMENTAL}

Several LAAPDs were investigated. All diodes were of the windowless, UV-enhanced type, produced recently by Advanced Photonix. Three of them are of $16 \mathrm{~mm}$ diameter and one of $10 \mathrm{~mm}$ diameter. Table 1 lists the main characteristics for each diode according to its data sheet.

The LAAPD was operated in a light-tight box to shield it from ambient light. The LAAPD signals were fed through a low-noise charge preamplifier (Canberra 2004, with a sensitivity of $45 \mathrm{mV} \mathrm{MeV}^{-1}$ ) to a spectroscopy amplifier (HP5582A, with a gain of 32-256) and sorted in a 1024channel multi-channel analyser (Nucleus PCA II). For pulse-amplitude and energy-resolution measurements, the x-ray pulse-height distributions were fitted to a Gaussian function superimposed on a linear background, from which the centroid and the full width at half-maximum were determined.

\section{OPERATIONAL CHARACTERISTICS}

The detector characteristics were evaluated with $5.9 \mathrm{keV} \mathrm{Mn}$ $\mathrm{K} \alpha$ x-rays from a ${ }^{55} \mathrm{Fe}$ source. The $6.4 \mathrm{keV} \mathrm{Mn} \mathrm{K} \beta$ x-rays were removed by absorption in a chromium filter. These characteristics included determinations of the optimum LAAPD operating voltage and gain, energy resolution, dark current effect, spatial uniformity, lowest detectable x-ray energy and count rate capability. The incident x-ray flux was limited to few thousand photons per second except for the count-rate behaviour study. Optimum shaping time

Table 1. LAAPD parameters just before breakdown, from the data sheets

\begin{tabular}{ccccc}
\hline $\begin{array}{c}\text { LAAPD } \\
\text { No. }\end{array}$ & $\begin{array}{c}\text { Diameter } \\
(\mathrm{mm})\end{array}$ & $\begin{array}{c}\text { Voltage } \\
(\mathrm{V})\end{array}$ & Gain & $\begin{array}{c}\text { Dark current } \\
(\mathrm{nA})\end{array}$ \\
\hline 1 & 16 & 1840 & 317 & 201 \\
2 & 16 & 1851 & 312 & 336 \\
3 & 16 & 1849 & 303 & 499 \\
4 & 10 & 1873 & 314 & 222 \\
\hline
\end{tabular}

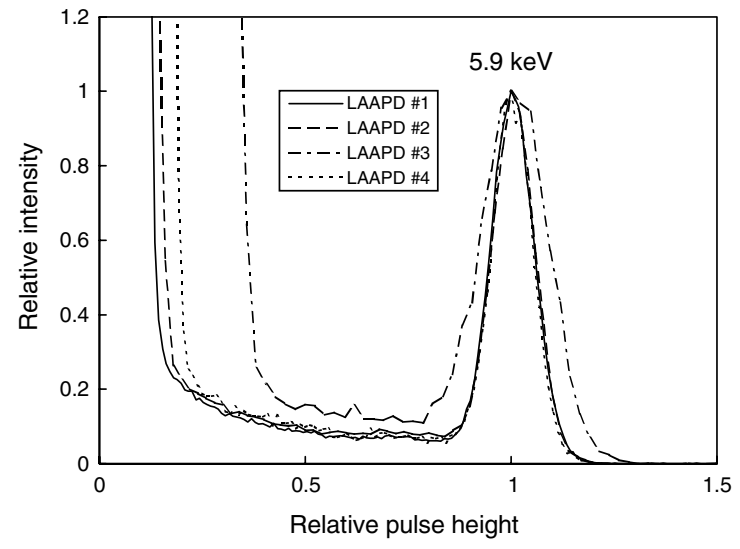

Figure 2. Typical pulse-height distributions for the LAAPDs used in this work for $5.9 \mathrm{keV} \mathrm{x}$-rays, illustrating the low-energy-noise-tail limit.

constants used in the amplifier were found to be about $0.1-0.2 \mu \mathrm{s}$.

Figure 2 shows a typical pulse-height distribution for the $5.9 \mathrm{keV}$ x-rays for the different photodiodes. The peak departs from a Gaussian shape, presenting a tail towards the low-energy region. This tail results from $\mathrm{x}$-ray interactions deep within the photodiode multiplication region (Fig. 1), generating pulses that are only partially amplified, resulting in reduced pulse amplitudes. ${ }^{1}$ Similarly, x-ray interactions that occur in the weak electric field of the undepleted $\mathrm{p}$ region may result in primary electrons being lost to the front $\mathrm{p}^{+}$contact or to traps, again leading to smaller amplitude pulses. ${ }^{1}$ The electronic noise tail in the low-energy limit depends on the photodiode dark current and sets a limit to the lowest detectable x-ray energy.

\section{LAAPD gain}

Figure 3 depicts the detector gain, energy resolution and low-energy noise tail limit as a function of LAAPD 4 bias voltage, for $5.9 \mathrm{keV} x$-rays. The relative pulse amplitude was normalized to the value obtained at $1400 \mathrm{~V}$, corresponding to a gain of 3.5 according to the manufacturer. The variation

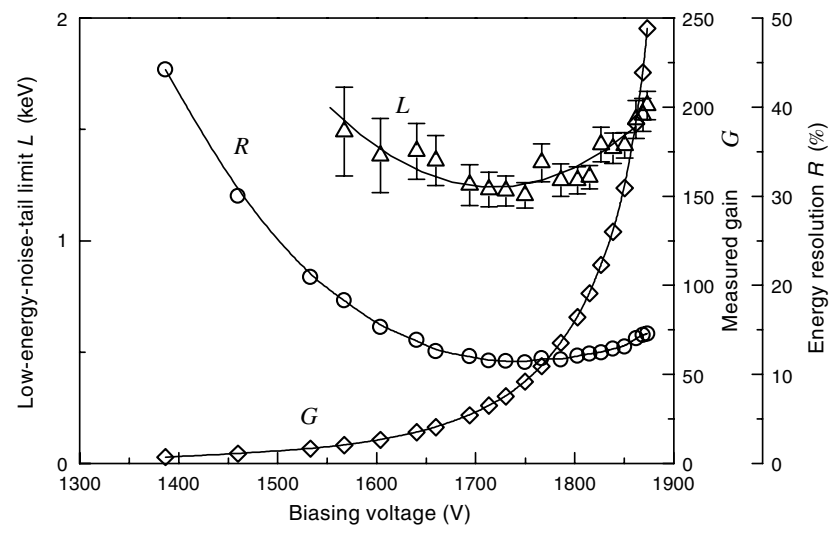

Figure 3. LAAPD 4 relative pulse amplitude, energy resolution and low-energy noise tail limit as a function of reverse bias voltage, for $5.9 \mathrm{keV}$ x-rays. Relative pulse amplitude and energy resolution experimental uncertainties fall within the symbol size. 
Table 2. Optimum performance characteristics for the LAAPDs in this work, for $5.9 \mathrm{keV}$ x-rays

\begin{tabular}{ccccc}
\hline $\begin{array}{c}\text { LAAPD } \\
\text { No. }\end{array}$ & $\begin{array}{c}\text { Measured } \\
\text { gain }\end{array}$ & $\begin{array}{c}\text { Low-energy noise } \\
\text { tail limit }(\mathrm{keV})\end{array}$ & $\begin{array}{c}\text { Energy resolution } \\
(1 \mathrm{~mm} \text { collimation) }(\%)\end{array}$ & $\begin{array}{c}\text { Energy resolution } \\
\text { (full area) (\%) }\end{array}$ \\
\hline 1 & 72 & 0.9 & 10.3 & 12.3 \\
2 & 53 & 1.1 & 11.8 & 14.9 \\
3 & 52 & 2.2 & 17.9 & 18.8 \\
4 & 46 & 1.2 & 11.4 & 12.3 \\
\hline
\end{tabular}

of detector gain is in good agreement with the exponential gain of the LAAPD specified by the manufacturer. However, the best detector energy resolution is achieved for gains of only 50-70 and degrades with gain at higher values. At these gains, the low-energy noise tail limit has already stabilized around its minimum and increases slightly at higher gains. Table 2 presents the best energy resolutions, low-energy noise tail limit and gains obtained for the different LAAPDs for both $1 \mathrm{~mm}$ diameter and full-area $\mathrm{x}$-ray beam illumination.

\section{Dark current effects}

Figure 2 and Tables 1 and 2 illustrate the effect of the dark current on LAAPD performance. Higher dark currents result in reduced performance for both detector energy resolution and lowest detectable $x$-ray energy. The degradation in detector energy resolution with detector bias voltage, for gains above $\sim 50-70$, as shown in Fig. 3, is mainly due to the greater increase in dark current. ${ }^{10}$ The optimum operating gain depends on the individual LAAPD, and a compromise between gain and dark current has to be obtained for each one.

We note that all the $16 \mathrm{~mm}$ diameter LAAPDs used in this work are within the manufacturer's specifications for a maximum dark current of $600 \mathrm{nA}$ at a gain of 200. Still, photodiode performance can be significantly different.

\section{Spatial uniformity}

Non-uniform material resistivity of the LAAPD results in gain fluctuations due to localized electric field variations. Thus, the LAAPD response can vary with the $x$-ray incidence point on the LAAPD surface. Spatial uniformity was studied using a $0.5 \mathrm{~mm}$ collimated x-ray beam positioned at 17 equally distributed points over the area of the photodiode: one point at its center and eight points at radial distances of 3 and $6.5 \mathrm{~mm}$ for the $16 \mathrm{~mm}$ diameter LAAPDs.

The distributions of the gains of LAAPDs 2 and 3, normalized to the average value, are given in Table 3. A $\pm 4 \%$ variation of the $x$-ray pulse amplitude due to nonuniformities was observed. For LAAPD 2, only 50\% of the amplitudes are within $\pm 1.5 \%$ of the average. The same trend was noted for the different LAAPDs and the results do not suggest any correlation between dark current and non-uniformity. On the other hand, detector non-uniformity increases with increasing gain. This effect is related to the photodiode bias voltage increase, which results in larger local differences of the electric field within the depletion region, leading to larger differences on the avalanche multiplication gain.
Table 3. Distributions of pulse amplitudes normalized to the average value for a $0.5 \mathrm{~mm}$ collimated $5.9 \mathrm{keV}$ x-ray beam, measured at 17 points, equally distributed over the photodiode area: one point at its center and 8 points at radial distances of 3 and $6.5 \mathrm{~mm}$ for both LAAPD 2 and LAAPD 3

\begin{tabular}{cccccccccc}
\hline \multicolumn{1}{c}{ Normalised amplitude } \\
\hline $\begin{array}{c}\text { LAAPD } \\
\text { No. }\end{array}$ & 0.96 & 0.97 & 0.98 & 0.99 & 1.00 & 1.01 & 1.02 & 1.03 & 1.04 \\
\hline 2 & 1 & 1 & 3 & 6 & 1 & 2 & 1 & 2 & 0 \\
3 & 1 & 1 & 0 & 6 & 4 & 2 & 1 & 0 & 2 \\
\hline
\end{tabular}

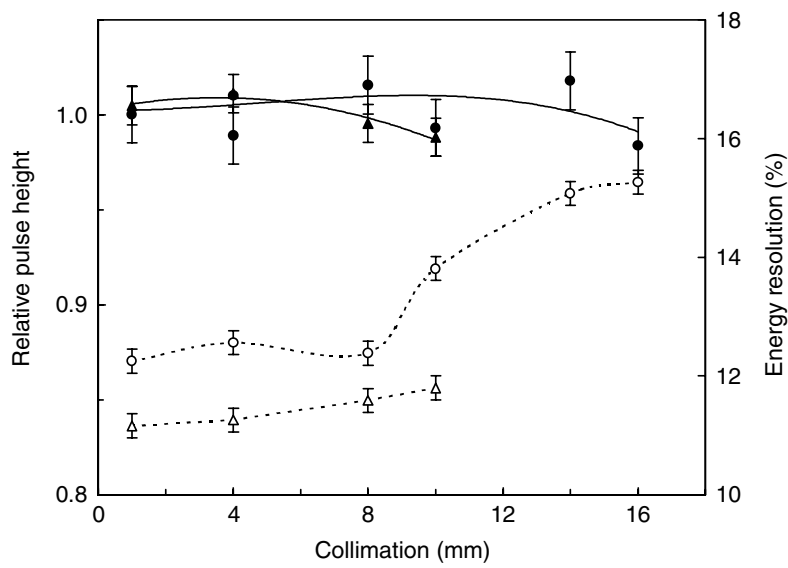

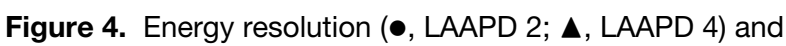
relative amplitude (O, LAAPD 2; $\triangle$, LAAPD 4) as a function of the $\mathrm{x}$-ray collimation for $5.9 \mathrm{keV} \mathrm{x}$-rays.

As a consequence of the LAAPD non-uniformity, energy resolution degrades with the collimating diameter at the front of the device. In Fig. 4, the detector energy resolution is presented as a function of the x-ray collimation for LAAPDs 2 and 4. A small degradation of the energy resolution is observed, reflecting the small spatial non-uniformity of the diodes.

\section{Count rate capability}

The detector pulse amplitude and energy resolution as a function of count rate was investigated for collimated $5.9 \mathrm{keV}$ $\mathrm{x}$-ray beams, $8 \mathrm{~mm}$ in diameter, for LAAPD 4 . Figure 5 presents the results obtained for LAAPD 4 . Count rates of about $4 \times 10^{4}$ counts $^{-1}$ are possible without significant degradation of LAAPD performance. At this count rate, a reduction of $3 \%$ in the pulse amplitude and a degradation from $12 \%$ to $13 \%$ in the energy resolution were measured. This behaviour results from the short pulse duration (a few 


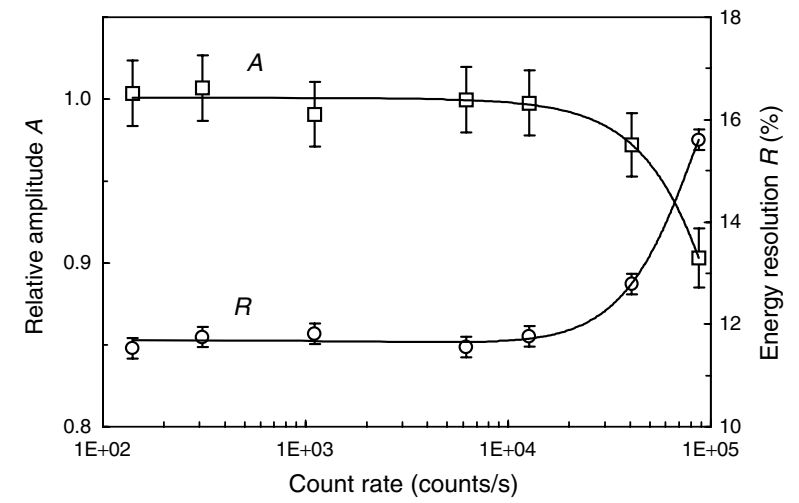

Figure 5. LAAPD 4 relative pulse amplitude and energy resolution as a function of count rate for $8 \mathrm{~mm}$ diameter collimated $5.9 \mathrm{keV} \mathrm{x}$-rays.

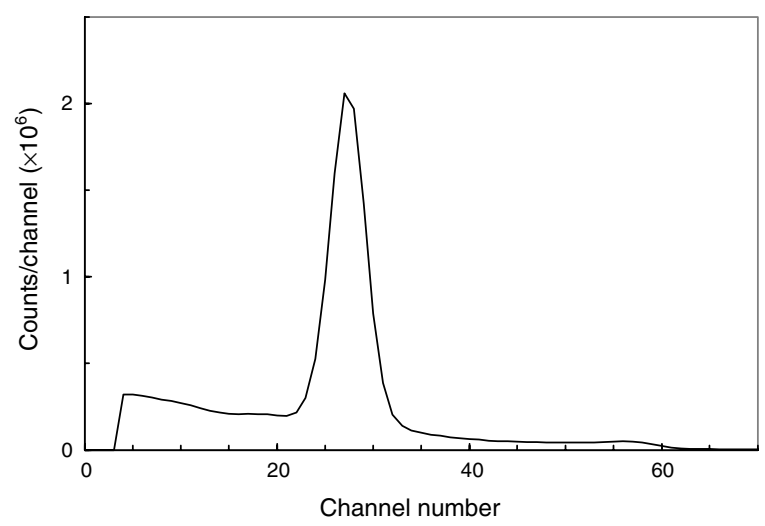

Figure 6. Typical pulse-height distribution for $8 \times 10^{4}$ counts $\mathrm{s}^{-1}$ count rate, for $8 \mathrm{~mm}$ diameter collimated $5.9 \mathrm{keV}$ $\mathrm{x}$-rays.

hundred nanoseconds), making it possible to count at rates as high as $10^{5}$ counts $\mathrm{s}^{-1}$ without significant degradation of the pulse-height distributions. Figure 6 presents the measured pulse-height distribution of $\mathrm{x}$-rays from a ${ }^{55} \mathrm{Fe}$ source for a count rate of $8 \times 10^{4}$ counts $\mathrm{s}^{-1}$ and a shaping time of $0.1 \mu \mathrm{s}$. No distortions of the peak shape are observed except for the small tail due to pulse pile-up. The same trend was observed for LAAPD 2.

\section{Space charge effects}

Space charge effects can be caused by high signal current densities produced by either high-energy x-rays and/or high gains, resulting in a decrease in the electric field and local heating in the avalanche region (M. Moszynski, M. Szawlowski, M. Kapusta, M. Balcerzyk and D. Wolski, presented at the 1999 IEEE Nuclear Science Symposium, 24-30 October, Seattle, WA, USA). These result in a nonlinear response of the LAAPD.

We investigated the detector linearity by comparing the LAAPD pulse amplitudes for 5.9 and $22.1 \mathrm{keV}$ x-rays as a function of gain. The results obtained are depicted in Fig. 7. The 22.1-to-5.9 keV pulse amplitude ratio decreases with increasing gain. However, this effect is negligible for gains below 100, and is only noticeable for gains above 150. A decrease of $<1 \%$ is found for gains up to 100 , whereas for

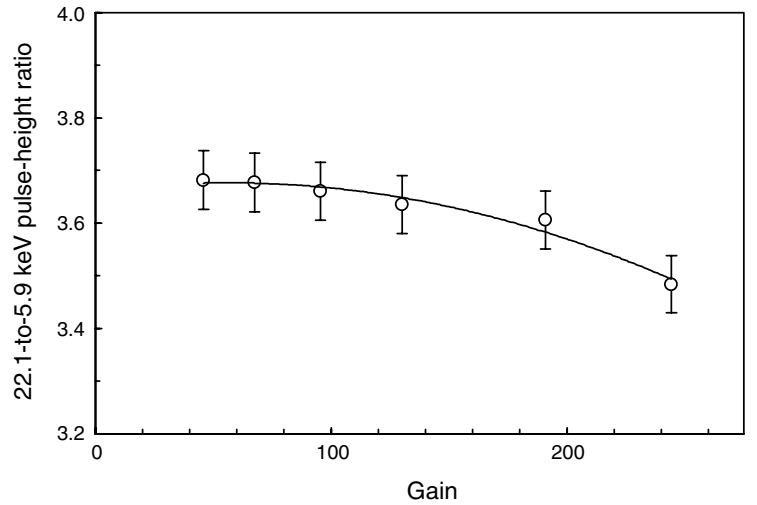

Figure 7. $22.1 / 5.9 \mathrm{keV} x$-ray pulse amplitude ratio as a function of LAAPD 4 gain.

gains of about 200 and 250 this decrease is about 3 and $6 \%$, respectively.

\section{Temperature dependence}

LAAPD gain and dark current depend significantly on temperature. ${ }^{8,10}$ Dark current can be reduced by about one order of magnitude and the minimum detectable energy can be reduced by a factor of two when the LAAPD temperature is reduced from room-temperature to $0{ }^{\circ} \mathrm{C} .{ }^{8}$ LAAPDs with built-in Peltier cooling devices for controlled-temperature operation are commercially available (Advanced Photonix). During our experiments, room temperature was stabilized at $20 \pm 0.3{ }^{\circ} \mathrm{C}$, and gain fluctuations of $<1 \%$ were observed.

\section{X-RAY SPECTROMETRY APPLICATIONS}

\section{Energy linearity and resolution}

The energy linearity and energy resolutions of the LAAPDs were determined in the $2-25 \mathrm{keV} x$-ray energy range using the fluorescent radiation induced in single-element samples ( $\mathrm{Si}, \mathrm{S}, \mathrm{Cl}, \mathrm{Ca}, \mathrm{Ti}, \mathrm{Cr}, \mathrm{Mn}, \mathrm{Fe}, \mathrm{Ni}, \mathrm{Zn}, \mathrm{As}, \mathrm{Se}, \mathrm{Rb}, \mathrm{Nb}$ and $\mathrm{Cd}$ ) and using direct irradiation of the detector with ${ }^{55} \mathrm{Fe}$ and ${ }^{109} \mathrm{Cd} \mathrm{x}$-ray sources. The measurement geometries are depicted to scale in Fig. 8 for direct irradiation (a) and for fluorescence measurements (b). To delimit the incident $x$-ray beam, 1 and $5 \mathrm{~mm}$ lead collimators were used for (a) and (b), respectively.

LAAPD 4 was used for this study and was operated in the optimum gain region with total count rates of the order of $10^{3}-10^{4}$ counts $\mathrm{s}^{-1}$. The centroids and relative full width at half-maximum values of the pulse-height distributions are plotted in Fig. 9 as a function of x-ray energy. Good energy linearity is observed throughout the energy range.

The detector energy resolution does not demonstrate a linear dependence on $E^{-1 / 2}$ but decreases slowly in the highenergy range limit. This behaviour can be attributed to peak distortion due to an increasing number of x-ray interactions in the multiplication region. Fig. 10 plots the LAAPD energy resolution as a function of $E^{-1 / 2}$. For comparison, a typical energy resolution $(14 \%$ at $6 \mathrm{keV})$ of a proportional counter is also depicted. For $x$-ray energies below $4 \mathrm{keV}$, the LAAPD energy resolution is worse than the $\mathrm{PC}$. Also, in the highenergy limit above $17 \mathrm{keV}$, the energy resolution tends to be higher than the PC. 


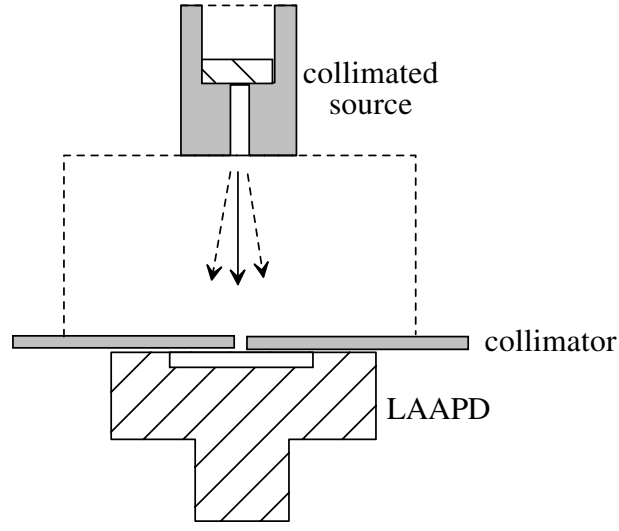

(a)

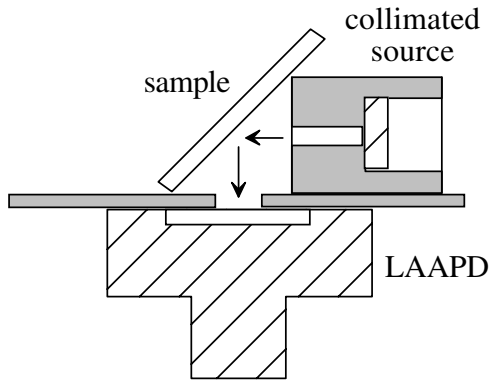

(b)

Figure 8. Set-up used for the measurements: (a) direct x-ray source irradiation; (b) fluorescence x-rays.

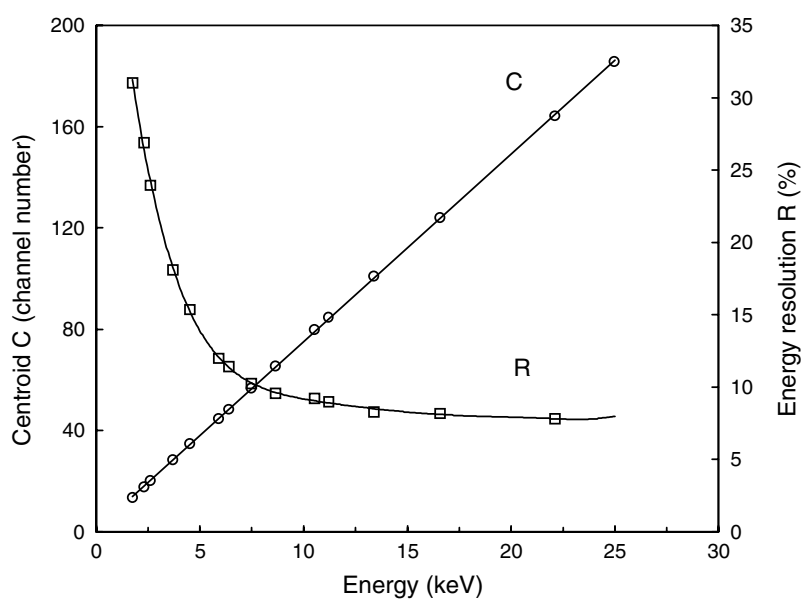

Figure 9. Peak centroid and energy resolution as a function of $\mathrm{x}$-ray energy for LAAPD 4 . The experimental uncertainties fall within the symbol size.

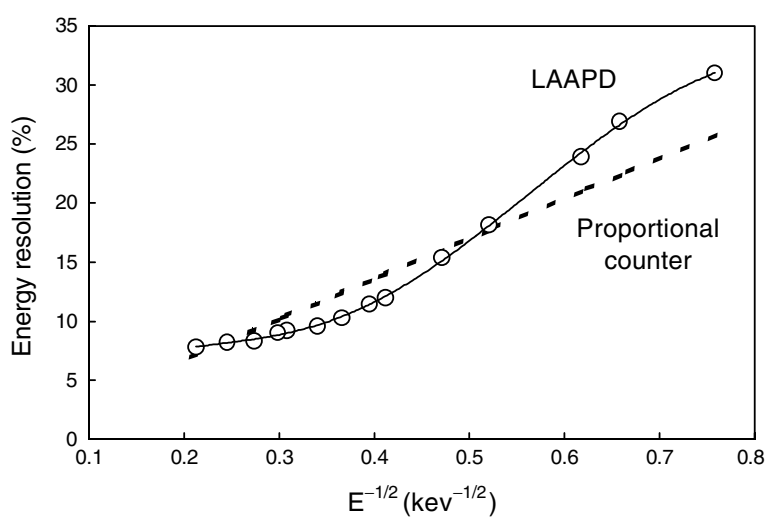

Figure 10. LAAPD energy resolution as a function of $E^{-1 / 2}$. The dashed line is the typical energy resolution of a proportional counter $(\mathrm{PC})$ with an energy resolution of $14 \%$ at $6 \mathrm{keV}$. The experimental uncertainties fall within the symbol size.

A typical x-ray spectrum obtained for the ${ }^{109} \mathrm{Cd}$ source is presented in Fig. 11 for an applied reverse bias voltage of $1750 \mathrm{~V}$ in the LAAPD at a count rate of 500 counts $\mathrm{s}^{-1}$ for a period of $500 \mathrm{~s}$.

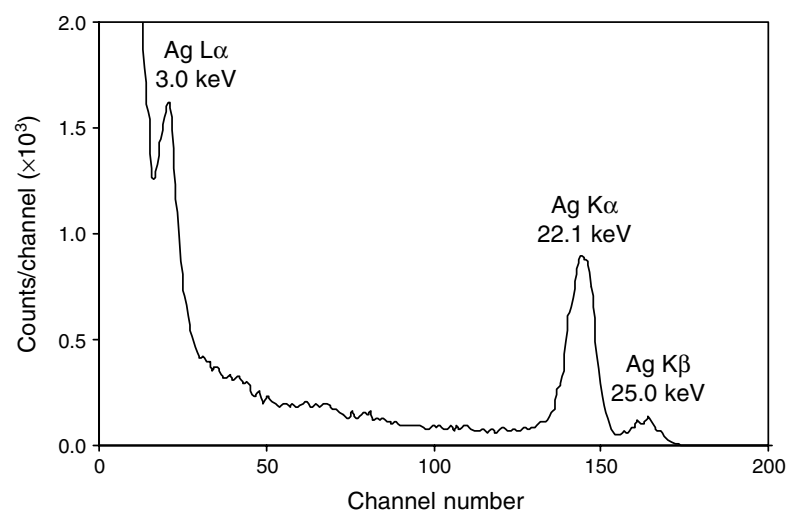

Figure 11. Typical $\mathrm{x}$-ray spectrum obtained for the ${ }^{109} \mathrm{Cd}$ source.

\section{Multi-element target samples}

Using the same geometry, $x$-ray fluorescence spectra were measured for thick samples of gypsum $\left(\mathrm{CaSO}_{4}\right)$, nonhomogeneous pyrite/chalcopyrite and SAES ST707 getter $(70 \% \mathrm{Zr}, 5.4 \% \mathrm{Fe}$ and $24.6 \% \mathrm{~V})$, excited with a ${ }^{109} \mathrm{Cd}$ x-ray source. The resulting pulse-height distributions are depicted in Fig. 12(a), (b) and (c), respectively. Spectral features include the elemental K-lines, the source backscattered radiation and the $\mathrm{Pb}$ L-lines from the source shielding and collimator [Fig. 12(b) and (c)]. The spectra are similar to those achieved with a conventional proportional counter, except for the soft x-ray region, where the windowless LAAPD detector can have an advantage if the device dark current is low enough to allow the detection of the x-ray pulses above the low-energy noise tail.

\section{DISCUSSION}

The performance characteristics of the room-temperature response of several LAAPDs to x-rays have been investigated. The results obtained demonstrate the applicability of LAAPDs to $x$-ray spectrometry in the $2-25 \mathrm{keV}$ range. The optimum detector operating gain, the influence of the dark current on the detector performance, the uniformity of the spatial response and the count rate capability were evaluated. Energy resolution and linearity and also space charge effects were determined for $x$-rays in the range $2-25 \mathrm{keV}$. 

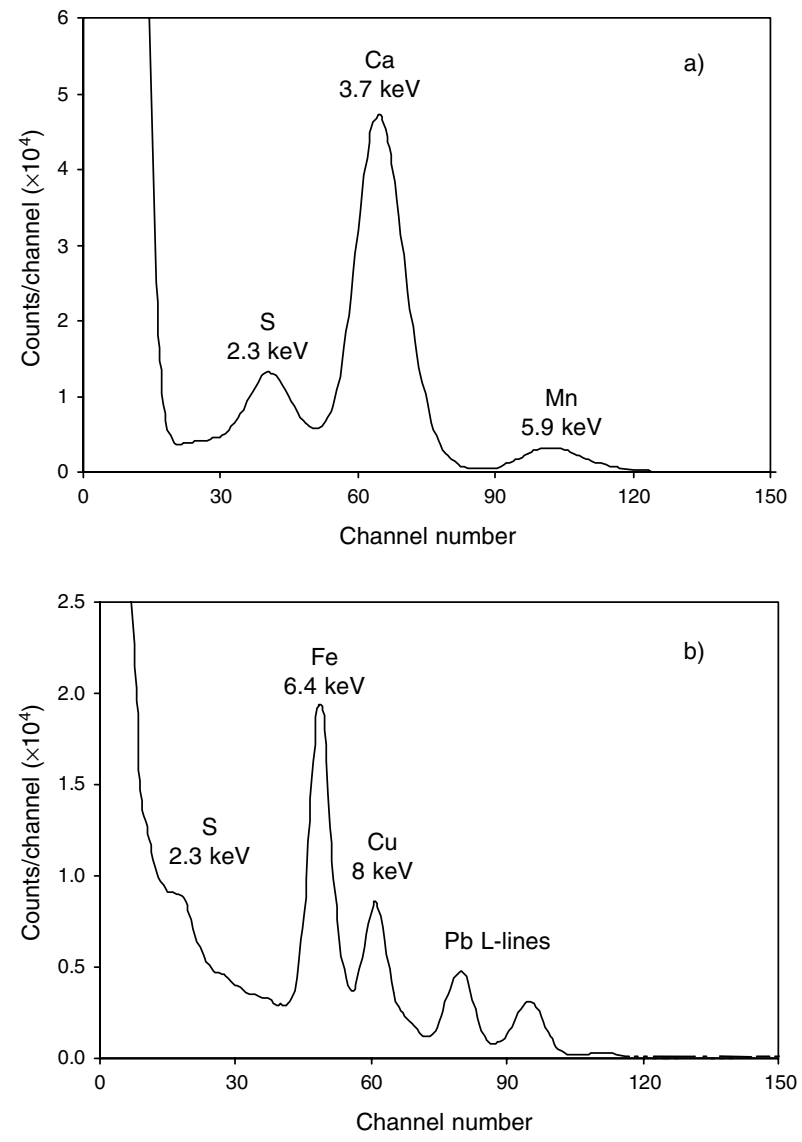

Figure 12. X-ray fluorescence spectra obtained with a ${ }^{109} \mathrm{Cd}$ $x$-ray source for thick samples of (a) gypsum $\left(\mathrm{CaSO}_{4}\right)$; (b) non-homogeneous pyrite/chalcopyrite; (c) SAES ST707 getter (70\% Zr, 5.4\% Fe and 24.6\% V).

Compared with proportional counters, the LAAPD is more compact and may provide improved energy resolutions for x-ray energies from a few up to about $20 \mathrm{keV}$. However, both energy resolution and minimum detectable $x$-ray energy depend on the LAAPD dark current, and LAAPDs within the manufacturer's specifications may present significantly different performances. For x-ray spectrometric applications, LAAPDs with low dark currents are desirable in order to obtain better energy resolutions than proportional counters.

The LAAPD cost, limited area, limited detection efficiency for medium- and high-energy $x$-rays, and

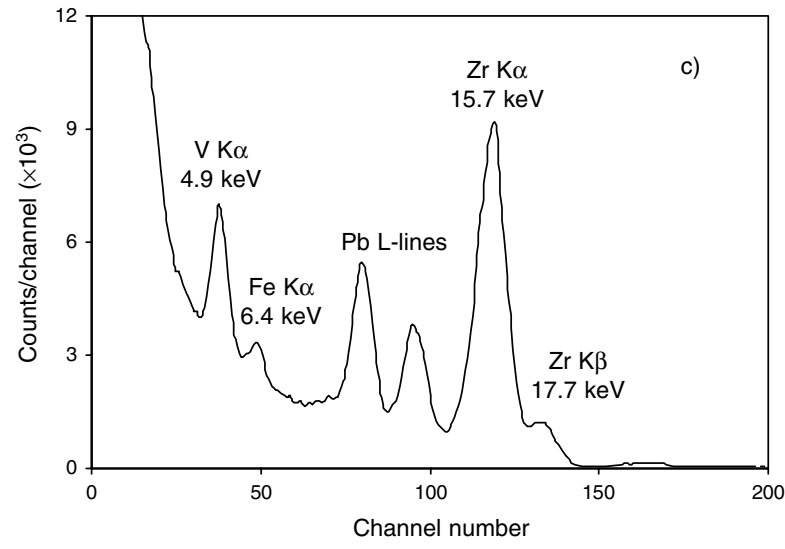

Figure 12. (continued).

sensitivity to light and temperature are drawbacks compared with proportional counters. However, its windowless feature may be crucial for the detection of soft x-rays. Additionally, its superior count-rate capability may be crucial for applications at high count rates.

\section{Acknowledgements}

The work was carried out in the Atomic and Nuclear Instrumentation Group of the Instrumentation Centre (Unit 217/94) of Departamento de Física, Universidade de Coimbra. Support is acknowledged from project CERN/P/FIS/15200/99.Thanks are due to R. E. Morgado, Los Alamos National Laboratory (LANL), Los Alamos, NM, USA, for revision of the manuscript.

\section{REFERENCES}

1. Baron AQR, Ruby SL. Nucl. Instrum. Methods A 1994; 343: 517.

2. Gullikson EM, Gramsch E, Szawlowski M. Appl. Opti. 1995; 34: 4662.

3. Pansart JP. Nucl. Instrum. Methods A 1997; 387: 186.

4. Farrell R, Olschner F, Frederick E, McConchie L, Vanderpuye K, Squillante MR, Entine G. Nucl. Instrum. Methods A 1990; 288: 137.

5. Lopes JAM, dos Santos JMF, Conde CAN. Nucl. Instrum. Methods A 2000; 454: 421.

6. Farrel R, Vanderpuye K, Entine G, Squillante MR. IEEE Trans. Nucl. Sci. 1991; 38: 144

7. Webb PP, McIntyre RJ. IEEE Trans. Nucl. Sci. 1976; 23: 138.

8. Ochi A, Nishi Y, Tanimori T, Nucl. Instrum. Methods A 1996; 378: 267.

9. Allier CP, Valk H, Bom VR, Hollander RW, van Eijk CWE. IEEE Trans. Nucl. Sci. 1998; 45: 576.

10. LAAPD Application Notes. Advanced Photonix, Camarillo, CA, 1999. 